\section{Neuzulassung im MS-Bereich}

Die Europäische Kommission hat die Zulassung für Daclizumab (Zinbryta ${ }^{\circledR}$ ) zur Behandlung von erwachsenen Patienten mit schubförmiger Multipler Sklerose (RRMS) erteilt. Mit Daclizumab steht nun eine innovative Option mit einem in der MS-Therapie neuen Wirkprinzip zur Verfügung. Daclizumab ist ein humanisierter monoklonaler Antikörper (IgG1), der selektiv an die alpha-Untereinheit (CD25) des hochaffinen Interleukin-2-Rezeptors (IL-2R) auf aktivierten T-Zellen bindet. Der Wirkmechanismus des Immunmodulators besteht vermutlich in der Blockade der Aktivierung autoreaktiver T-Zellen, einem Hauptverursacher der Entzündungen im zentralen Nervensystem bei MS. Daclizumab führt zu einer Erhöhung der $\mathrm{CD} 6_{\text {bright }}$-NK-Zellen, die selektiv die Zahl aktivierter T-Zellen verringern können, ohne eine generelle Immunzelldepletion $z u$ verursachen. Daclizumab wird einmal monatlich als subkutane Selbstinjektion mit einer Fertigspritze appliziert. Es wurde gemeinsam von Biogen und AbbVie entwickelt.

Nach Informationen von Biogen

\section{Duloxetin generisch}

Depressionen, generalisierte Angststörungen und Schmerzen bei diabetischer Polyneuropathie sind neurologische Erkrankungen, die die Lebensqualität der Betroffenen auf beruflicher und sozialer Ebene in hohem Maß beeinträchtigen. Mit Duloxetin Glenmark $30 \mathrm{mg}$ und $60 \mathrm{mg}$ steht ab sofort eine qualitätsvolle und preiswerte Therapieoption für Patienten mit diesen Krankheitsbildern zur Verfügung.

Nach Informationen von Glenmark

\section{Epilepsieportfolio erweitert}

Seit Juli ist das Epilepsieportfolio von Desitin um die Substanz Zonisamid unter dem Namen Desizon ${ }^{\circledast}$ in drei verschiedenen Wirkstärken erweitert. Es ist indiziert als Monotherapie für die Behandlung von fokalen Epilepsien mit oder ohne sekundäre Generalisierung bei Erwachsenen sowie als Zusatztherapie bei fokalen Epilepsien mit oder ohne sekundäre Generalisierung bei Erwachsenen, Jugendlichen und Kindern ab sechs Jahren.

Nach Informationen von Desitin
Galenus-von-Pergamon-Preis 2016

\section{Das Antidepressivum unter den Bewerbern}

— Vortioxetin (Brintellix ${ }^{\oplus}$ ) von Lundbeck ist ein multimodal wirkendes Antidepressivum zur Therapie von Episoden einer Major Depression bei Erwachsenen. Über seine antidepressive Wirksamkeit hinaus hat es positive Effekte auf kognitive Funktionen und Alltagsfähigkeiten, die bei einer Depression meist beeinträchtigt sind.

Vortioxetin ist nicht nur ein Serotonin-Wiederaufnahmehemmer, sondern moduliert zusätzlich die Funktion einer Reihe weiterer prä- und postsynaptischer Serotoninrezeptoren, sodass in verschiedenen Systemen des Gehirns erhöhte Neurotransmitterkonzentrationen vorliegen. Auf diesen Wirkmechanismus wird die neben der antidepressiven Wirkung erstmals in einer kontrollierten Studie nachgewiesene Verbesserung kognitiver Funktionen zurückgeführt.

Es werden zwei komplementäre Wirkmechanismen angenommen: eine Hemmung der Wiederaufnahme von Serotonin (5-HT) sowie direkte Aktivitäten an verschiedenen 5-HT-Rezeptoren (Abb. 1). Außerdem hemmt der Wirkstoff den präsynaptischen 5-HT-Transporter. In präklinischen Untersuchungen konnten für Vortioxetin antagonistische Wirkungen an den Rezeptoren $5-\mathrm{HT}_{3}, 5-\mathrm{HT}_{7}$ und $5-\mathrm{HT}_{1 \mathrm{D}}$, partiell agonistische Wirkungen an den $5-\mathrm{HT}_{1 \mathrm{~B}}$-Rezeptoren sowie agonistische Wirkungen an den $5-\mathrm{HT}_{1 \mathrm{~A}}-$ Rezeptoren gezeigt werden (Abb. 1). Dies führt zur Modulation der Neurotransmission in verschiedenen Systemen, vorrangig des Serotoninsystems, wahrscheinlich aber auch des Noradrenalin-, Dopamin-, Histamin-, Acetylcholin- sowie

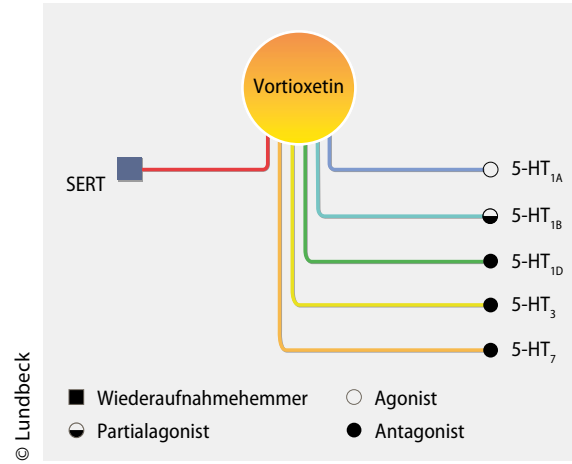

Multimodales Wirkprofil von Vortioxetin

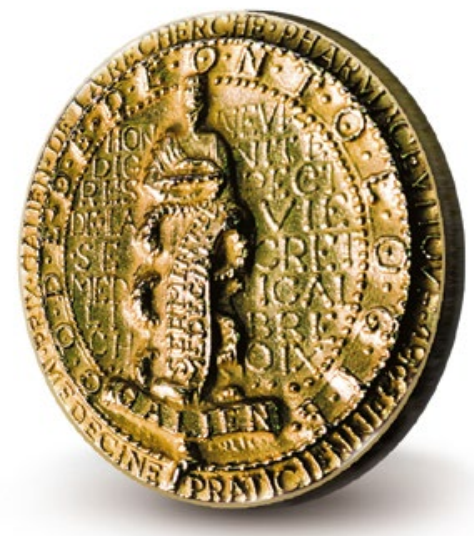

Mit dem von Springer Medizin gestifteten Galenus-von-Pergamon-Preis werden jedes Jahr Arzneimittelinnovationen ausgezeichnet. Einer der 15 Bewerber in 2016 ist das Antidepressivum Vortioxetin (Brintellix ${ }^{\circledR}$.

des GABAergen und des glutamatergen Systems.

Klinische Wirksamkeit und Sicherheit von Vortioxetin sind in einem Studienprogramm mit mehr als 6.700 Patienten ermittelt worden. In zwölf placebokontrollierten Kurzzeitstudien über jeweils sechs bis acht Wochen waren 3.700 Patienten mit akuten Episoden einer Major Depression behandelt worden. In neun der zwölf Studien war die Wirksamkeit in mindestens einer Dosisgruppe durch eine Differenz zu Placebo von mindestens zwei Punkten des MADRSSummenscores (Montgomery und Åsberg Depression Rating Scale) oder der HAMD24-Skala (Hamilton Depression Rating Scale 24 Item) nachgewiesen worden. Außerdem waren signifikante Effekte bei der Verbesserung des klinischen Gesamteindrucks (Clinical Global Impression - Improvement Score, CGI-I) beobachtet worden.

In einer placebokontrollierten, randomisierten Studie $(n=602)$ konnte erstmals nachgewiesen werden, dass Vortioxetin innerhalb von acht Wochen statistisch signifikant die kognitive Performance von Patienten im Alter unter 65 Jahren mit rezidivierenden Episoden einer Major Depression verbessert. Dabei handelt es sich überwiegend um einen direkten Effekt auf die Kognition und nur zu einem geringen Teil um eine indirekte Folge aufgrund der antidepressiven Wirkung.

Vortioxetin steht als einmal täglich einzunehmende Filmtablette in Dosisstärken von $5 \mathrm{mg}, 10 \mathrm{mg}$ und $20 \mathrm{mg}$ sowie als Tropfen (20 mg/ml) zur Verfügung. 